\title{
Prevalência de dislipidemias e consumo alimentar: um estudo de base populacional
}

\author{
Prevalence of dyslipidemias and food consumption: \\ a population-based study
}

Silvia Eugênia Oliveira Valença (https://orcid.org/0000-0003-3592-9867) ${ }^{1}$

Alice Divina Melo Brito (https://orcid.org/0000-0002-6698-4802) ${ }^{1}$

Danielle Cristina Guimarães da Silva (https://orcid.org/0000-0001-5456-0853) ${ }^{2}$

Fabrícia Geralda Ferreira (https://orcid.org/0000-0001-9836-4176) ${ }^{3}$

Juliana Farias Novaes (https://orcid.org/0000-0003-3616-5096) ${ }^{1}$

Giana Zarbato Longo (https://orcid.org/0000-0001-7666-5007) ${ }^{4}$

${ }^{1}$ Universidade Federal de Viçosa. Av. Peter

Henry Rolfs s/n, Campus Universitário. 36570-

900 Viçosa MG Brasil. silviavalenca1@gmail.com

${ }^{2}$ Universidade Federal do

Oeste da Bahia. Barreiras

BA Brasil.

${ }^{3}$ Escola Preparatória de

Cadetes do Ar. Barbacena

MG Brasil.

${ }^{4}$ Universidade Federal

de Santa Catarina.

Florianópolis SC Brasil.

\begin{abstract}
This study aimed to assess the prevalence of dyslipidemia and its association with an adequate intake of carbohydrates, saturated, monounsaturated, trans, and omega-3 fats among adults living in Viçosa, Minas Gerais, Brazil. This is a cross-sectional study with 884 adults aged 20 to 59 years. Sociodemographic, food intake, anthropometric, and biochemical data were collected. Associations between study variables were investigated by the chi-square test. There was a high prevalence of dyslipidemia in the study population (64.25\%), with most individuals having abnormal levels of at least one serum lipid component. Inadequate intakes of saturated, trans, and monounsaturated fats and carbohydrates were predominant. It is noteworthy that omega-3 intake levels were adequate in most individuals. Eutrophic adults showed a higher prevalence of excessive intake of saturated and trans fats. It was found that $38.7 \%$ of individuals with low levels of High Density Lipoprotein cholesterol (HDL-c) had an excessive intake of saturated fat. Most individuals with high triglyceride levels or high triglyceride/HDL-c ratios had an insufficient intake of monounsaturated fat. Further studies are needed to evaluate other factors that may influence dietary patterns.
\end{abstract}

Key words Dyslipidemias, Eating, Adults, Nutritional epidemiology
Resumo O presente artigo objetivou estimar a prevalência de dislipidemias e sua associação $e$ adequação da ingestão de gorduras saturadas, monoinsaturadas, trans, ômega-3 e carboidratos, em adultos de Viçosa-MG. Trata-se de um estudo transversal com 884 adultos com idades entre 20 e 59 anos. Foram coletados dados sociodemográficos, de consumo alimentar, antropométricos e bioquímicos da população. As associações entre as variáveis foram verificadas utilizando-se o teste qui-quadrado. Verificou-se elevada prevalência de dislipidemias na população (64,25\%), com pelo menos um dos lipídeos séricos alterados. Houve predomínio de inadequação na ingestão de gorduras saturadas, trans, monoinsaturadas e carboidratos. Ressalta-se que a ingestão de ômega-3 esteve adequado para a maioria dos indivíduos. Adultos eutróficos apresentaram maiores prevalências de ingestão excessiva de gorduras saturadas e trans. Observou-se que 38,7\% dos indivíduos com HDL-c reduzido apresentaram ingestão acima do recomendado de gordura saturada. A maioria dos indivíduos com triglicerídeos e razão TG/HDL-c elevada possuíam ingestão insatisfatória de gordura monoinsaturada. Faz-se necessária a avaliação de outros fatores que podem influenciar o padrão alimentar.

Palavras-chave Dislipidemias, Ingestão de alimentos, Adultos, Epidemiologia nutricional 


\section{Introdução}

As dislipidemias são definidas como alterações na concentração plasmática das lipoproteínas (lipoproteína de baixa densidade - LDL-c; lipoproteína de alta densidade - HDL-c; e triglicerídeos -TG) ${ }^{1}$.Um aumento nas concentrações séricas de TG, redução nas concentrações de HDL-c e aumento das concentrações das LDL-c pequenas e densas são apontados como fatores de risco independentes para o desenvolvimento das doenças ateroscleróticas ${ }^{2}$. A doença aterosclerótica pode se agravar até a ocorrência de eventos coronarianos como infarto, angina e morte cardiovascular ${ }^{3}$.

Além dos danos causados na saúde do indivíduo durante os anos vividos com a doença, as dislipidemias contribuem para que as doenças ateroscleróticas constituam a primeira causa de óbitos no mundo, assim como no Brasil ${ }^{4,5}$. A taxa de mortalidade cardiovascular global devido à hipercolesterolemia é igual a 1,7 por 100.000 habitantes, enquanto no Brasil, essa mesma taxa equivalia a 2,5 óbitos por 100.000 habitantes no ano de $2010^{6}$. Segundo Marinho et al. ${ }^{7}$, o colesterol total elevado foi o oitavo fator de risco que mais contribuiu para a morbimortalidade de homens e mulheres no Brasil, e por isso, deve ser foco de políticas públicas.

A avaliação das dislipidemias como um indicador de saúde também é justificada pela sua alta prevalência na população brasileira, como demostrado em estudos observacionais de base populacional conduzidos, que revelaram prevalências de dislipidemia acima de $60 \% \%^{8,9}$ e $75 \%{ }^{10}$ ao se investigar indivíduos obesos. Além disso, pesquisas nacionais com diagnóstico de dislipidemia autorreferida também apresentaram prevalências preocupantes como a Pesquisa $\mathrm{Na}$ cional de Saúde (PNS) de $2013^{11}$ que apresentou uma prevalência de $12,5 \%$ na população acima de 18 anos e o inquérito da pesquisa de Vigilância de Fatores de Risco e Proteção para Doenças Crônicas por Inquérito Telefônico (VIGITEL) do ano de $2016^{12}$, que encontrou uma frequência de relato de diagnóstico médico de dislipidemia de $24,8 \%$, ao investigar as 26 capitais brasileiras e o Distrito Federal.

Logo, dada sua elevada frequência na população geral, a identificação precoce dos fatores de risco para a prevenção e tratamento pode ser o caminho para o controle efetivo das dislipidemias e a redução da mortalidade cardiovascular. Embora os determinantes das dislipidemias variem consideravelmente entre os indivíduos, é possível apontar os principais fatores envolvidos, tais como dieta, atividade física e herança genética ${ }^{13}$. Em relação ao consumo alimentar, as concentrações séricas de colesterol total e triglicerídeos se elevam em função da ingestão aumentada de colesterol, de carboidratos, ácidos graxos saturados e trans e de excessiva quantidade de calorias ${ }^{14}$. Esse padrão alimentar, considerado altamente aterogênico, pode contribuir em até $62 \%$ para a ocorrência de óbitos antes dos 70 anos na população brasileira ${ }^{15}$.

Observando a atual situação da população brasileira em relação à presença de dislipidemias e levando em consideração os efeitos agravantes de uma alimentação inadequada, o presente estudo teve como objetivo avaliar a prevalência de dislipidemia, sua associação e adequação da ingestão de gorduras saturadas, monoinsaturadas (MUFA), trans, ômega-3 e carboidratos em adultos do município de Viçosa-MG.

\section{Metodologia}

\section{Desenho do estudo}

Estudo transversal, de base populacional, com análise descritiva e analítica, utilizando-se o banco de dados do estudo intitulado "Síndrome metabólica e fatores associados: um estudo de base populacional em adultos de Viçosa-MG" realizado pelo grupo de Estudo em Saúde e Alimentação (ESA), conduzido entre 2012 e 2014, na cidade de Viçosa, Minas Gerais. A população do estudo incluiu indivíduos entre 20-59 anos, de ambos os sexos e residentes da área urbana do município.

$\mathrm{Na}$ primeira etapa do estudo foi realizada uma visita domiciliar com aplicação de um questionário estruturado que envolveu aspectos socioeconômicos e demográficos. Já na segunda etapa foi aplicado um Questionário de Frequência de Consumo Alimentar (QFCA) validado para a população de Viçosa ${ }^{16}$, realizada a avaliação antropométrica e a coleta de sangue.

\section{Cálculo do tamanho amostral e seleção dos participantes}

O tamanho amostral foi calculado utilizando o programa OpenEpi® ${ }^{\circledR}$, versão online 3.03a, considerando a população de referência pelo censo 2010 (43.431 indivíduos); prevalência esperada de $60,3 \%$; erro amostral de $4,5 \%$; efeito do desenho de 1,7 totalizando a necessidade mínima de 765 indivíduos. A este valor foi adicionado 10\% 
para perdas e 10\% para controle de variáveis de confusão, totalizando uma amostra estimada de 918 indivíduos.

A seleção dos indivíduos se deu por conglomerados, com amostragem de duplo estágio, sendo as unidades de primeiro estágio os setores censitários, unidades de recenseamento do Instituto Brasileiro de Geografia e Estatística (IBGE) e de segundo estágio, os domicílios. Foram sorteados 30 setores censitários dentre os 99 existentes na zona urbana de Viçosa-MG, posteriormente, sorteou-se o número de quarteirão e a esquina, iniciando-se o trabalho de campo no sentido horário da esquina sorteada. A metodologia se encontra detalhada em Segheto et al. ${ }^{17}$.

Foram excluídos do estudo as gestantes, indivíduos acamados ou impossibilitados de levantar-se para a realização das medidas antropométricas e aqueles com alguma incapacidade mental que comprometesse as respostas durante a entrevista.

\section{Aspectos socioeconômicos e demográficos}

A idade dos indivíduos foi selecionada como variável demográfica e categorizada em faixas etárias: 20-29; 30-39; 40-49 e 50-59 anos. Como variáveis socioeconômicas, utilizou-se anos de escolaridade e nível socioeconômico. A escolaridade foi descrita em anos de estudo e categorizada em $\leq 8$ anos de estudo, 9-11 anos de estudo, $\geq 12$ anos de estudo. O nível socioeconômico dos indivíduos foi classificado de acordo com o Critério Brasileiro de Classificação Econômica da Associação Brasileira de Empresas de Pesquisas $(\mathrm{ABEP})^{18}$, categorizado em nível alto (classes A1, A2, B1 e B2), médio (classes C1 e C2) e baixo (classes D e E).

\section{Avaliação Antropométrica e Bioquímica}

As seguintes medidas antropométricas foram coletadas: peso, altura e perímetro da cintura (PC). O peso $(\mathrm{Kg})$ foi obtido por uma balança portátil em escala antropométrica Tanita (com capacidade de $200 \mathrm{Kg}$ ) e a altura (m) foi medida utilizando-se um estadiômetro Welmy fixo, de haste de 2,5 metros de comprimento, acoplado à parede. Calculou-se o Índice de Massa Corporal (IMC) após as mensurações e os indivíduos foram classificados como excesso de peso (IMC $\geq 25$ $\mathrm{kg} / \mathrm{m}^{2}$ ) e peso normal (IMC $\left.\leq 24,9 \mathrm{~kg} / \mathrm{m}^{2}\right)^{19}$. O PC $(\mathrm{cm})$ foi aferido por fita métrica inelástica milimetrada com 1,5 metros de comprimento, no ponto médio entre a última costela e a crista ilía- ca, sendo considerada como elevada quando seu valor fosse $\geq 80 \mathrm{~cm}$ para mulheres e $\geq 90 \mathrm{~cm}$ para homens, demonstrando risco aumentado para as doenças cardiovasculares ${ }^{20}$.

Os voluntários realizaram a coleta sanguínea, com auxílio de um profissional registrado no Conselho Regional de Enfermagem. Foram repassadas orientações prévias para que os voluntários realizassem jejum de 12 horas e não alterassem seus hábitos de vida. Para as análises bioquímicas, o conteúdo sanguíneo foi armazenado em tubo a vácuo contendo gel separador com ativador de coágulos, e o material foi centrifugado durante 15 minutos a $3000 \mathrm{rpm}$ (2000 G). O colesterol total, triglicerídeos, HDL-c foram medidos com reagentes enzimáticos e quantificados fotometricamente usando um autoanalisador COBAS Mira Plus (Roche Diagnostics Systems). A concentração de LDL-c foi calculada usando a equação de Friedewald ${ }^{21}$. As amostras de sangue foram analisadas no setor de Divisão de Saúde da Universidade Federal de Viçosa.

Após a mensuração dos parâmetros bioquímicos, foram estabelecidas as razões CT/HDL-c, sendo considerada adequada uma razão $\leq 5$ para homens e $\leq 4,5$ para mulheres. O LDL-c/HDL-c de $\leq 3,5$ e $\leq 3,0$ respectivamente para homens e mulheres e TG/HDL-c $<4$ para ambos os se$\operatorname{xos}^{22,23}$.

O diagnóstico de dislipidemia se deu com base nos pontos de corte da Diretriz Brasileira de Dislipidemia e Prevenção da Aterosclerose atualizada como hipercolesterolemia isolada: aumento isolado do LDL-c (LDL-c $\geq 160 \mathrm{mg} / \mathrm{dL}$ ); hipertrigliceridemia isolada: aumento isolado dos triglicerídeos ( $\mathrm{TG} \geq 150 \mathrm{mg} / \mathrm{dL}$ ); hiperlipidemia mista: aumento do LDL-c (LDL-c $\geq 160 \mathrm{mg} / \mathrm{dL}$ ) e dos TG (TG $\geq 150 \mathrm{mg} / \mathrm{dL}$ ); HDL-c baixo: redução do HDL-c (homens < $40 \mathrm{mg} / \mathrm{dL}$ e mulheres $<50 \mathrm{mg} /$ $\mathrm{dL}$ ) isolada ou em associação ao aumento de LDL -c ou de $\mathrm{TG}^{24}$.

\section{Consumo alimentar}

O QFCA quantitativo desenvolvido incluiu informações sobre o consumo habitual de 95 itens alimentares, distribuídos em 26 grupos alimentares. A estimativa da ingestão de ácidos graxos saturados, trans, MUFA, ômega-3 e carboidratos verificada no QFCA foi realizada a partir da tabulação no software Brasil-Nutri, desenvolvido para a Pesquisa de Orçamentos Familiares (POF 2008-2009), realizada pelo IBGE ${ }^{25,26}$.

Para a quantificação desses nutrientes, elaborou-se uma planilha no programa Excel, versão 
2010 (Microsoft Corp., Estados Unidos), com base no cálculo: Quantidade de porções consumidas por vez X Peso/medida da porção X Frequência de consumo X Composição nutricional da porção do alimento para a estimativa do consumo diário. Aqueles indivíduos com consumo calórico estimado acima de $6000 \mathrm{Kcal}$ foram considerados outliers e foram excluídos das análises ${ }^{27}$.

A ingestão de gordura saturada foi classificada como inadequada quando a contribuição de calorias proveniente desse nutriente totalizou mais de $10 \%$ do valor calórico total da dieta (VCT), segundo a I Diretriz sobre o Consumo de Gorduras e Saúde Cardiovascular ${ }^{3}$. De acordo com a mesma diretriz, foi considerada inadequada a ingestão de gordura trans quando acima de $1 \%$ do VCT; para as gorduras monoinsaturadas, quando menor que $15 \%$ do VCT e para ácidos graxos ômega-3, menor que $1 \mathrm{~g} / \mathrm{dia}$.

Para avaliar a adequação da ingestão dietética de carboidrato, foi utilizada a RDA do Institute of Medicine Dietary Reference Intakes (DRIs) de 130 g por $\mathrm{dia}^{28}$.

\section{Análises estatísticas}

Para a análise dos dados, foi utilizado o software STATA versão 13.1, levando em consideração o efeito do delineamento amostral, pelo grupo de comandos "svy", o qual considera o efeito da expansão da amostra na análise dos dados. As análises foram ponderadas por sexo, idade e escolaridade, sendo os pesos determinados pela razão entre as proporções de indivíduos, segundo dados do IBGE e na amostra.

Todas as variáveis foram apresentadas categoricamente, por meio de frequências absolutas e relativas. Foi aplicado o teste do qui-quadrado de Pearson para avaliar a associação entre as variáveis. Considerou-se o nível de significância estatística de $5 \%(\alpha \leq 0,05)$.

\section{Questões éticas}

O projeto foi aprovado pelo Comitê de Ética em Pesquisa da Universidade Federal de Viçosa. Todos os participantes do estudo assinaram o termo do consentimento livre e esclarecido após explicação do objetivo do estudo.

\section{Resultados}

A amostra foi constituída por 884 indivíduos, sendo $52,31 \%$ do sexo masculino e com idade média de 37,7 anos. De acordo com os dados apresentados na Tabela 1, a maioria dos indivíduos pertencia a faixa etária de 20 e 29 anos $(31,80 \%)$ e $46,32 \%$ deles apresentaram excesso de peso. Os indivíduos dislipidêmicos apresentaram maior escolaridade, maior IMC e perímetro da cintura elevado em comparação aos indivíduos sem dislipidemia $(\mathrm{p}<0,001)$.

A prevalência de dislipidemias foi de $64,25 \%$. No entanto, não houve diferença estatística na adequação da ingestão de gorduras e carboidratos entre os indivíduos com e sem dislipidemia (Tabela 2).

As variáveis sociodemográficas, bioquímicas e clínicas foram comparadas levando em consideração a adequação da ingestão das gorduras saturadas, trans e carboidratos, de acordo com as diretrizes (Tabela 3). Os indivíduos com menor faixa etária (20-29 anos) apresentaram maior ingestão de gordura saturada $(\mathrm{p}=0,012)$ e trans $(\mathrm{p}<0,001)$ acima do recomendado. A escolaridade também esteve associada à maior inadequação de ingestão de gorduras saturadas $(\mathrm{p}<0,001)$ e trans $(p=0,009)$, uma vez que os indivíduos com mais de 12 anos de estudo apresentaram as maiores proporções de inadequação. Em relação ao nível socioeconômico, a maioria dos indivíduos com ingestão inadequada de gordura trans pertenceu à classe $\mathrm{C}(\mathrm{p}=0,027)$.

No que se refere ao aspecto antropométrico e bioquímico, aqueles com PC e razões CT/HDL e TG/HDL normais apresentaram maiores percentuais de ingestão de gordura saturada acima de $10 \%$ do VCT ( $\mathrm{p}=0,038 ; 0,025$ e 0,047 , respectivamente) e trans acima de $1 \%$ ( $\mathrm{p}=0,010 ; 0,033$ e 0,011 , respectivamente). Os que apresentaram HDL-c baixo também apresentaram ingestão elevada de gordura saturada $(\mathrm{p}=0,022)$ e aqueles com TG normais tiveram ingestão de gordura trans acima do recomendado $(\mathrm{p}=0,001)$. Finalmente, indivíduos com razão LDL/HDL elevada tiveram maior ingestão de gordura saturada, conforme observado na Tabela 3. A prevalência global de ingestão acima do recomendado para gordura saturada e trans nessa população, correspondeu a $61,2 \%$ e $74,2 \%$, respectivamente.

Exceto pelo CT $(\mathrm{p}=0,004)$ e a razão TG/HDL $(p=0,014)$, que foram inversamente proporcionais à ingestão de carboidratos, nenhuma outra variável esteve associada à ingestão elevada de carboidratos (Tabela 3). Apesar disso, quase o total da amostra apresentou ingestão inadequada de carboidratos $(99,1 \%)$, com uma média de ingestão de $343,30 \mathrm{~g}( \pm 14,24)$, acima do recomendado pela RDA que é de até $130 \mathrm{~g}$. 
Tabela 1. Características socioeconômica, demográfica e antropométricas, segundo a presença de dislipidemias na população adulta (20 a 59 anos: $n=884$ ) de Viçosa-MG, Brasil (2012/2014).

\begin{tabular}{|c|c|c|c|c|}
\hline \multirow{2}{*}{ Variáveis } & \multirow{2}{*}{ Total $(\%)$} & \multicolumn{2}{|c|}{ Dislipidemias } & \multirow{2}{*}{$\mathbf{p}^{*}$} \\
\hline & & $\operatorname{Sim}(\%)$ & Não (\%) & \\
\hline \multicolumn{5}{|l|}{ Sexo } \\
\hline Masculino & 52,31 & 32,56 & 19,75 & 0,263 \\
\hline Feminino & 47,69 & 34,09 & 13,61 & \\
\hline \multicolumn{5}{|l|}{ Faixa etária (anos) } \\
\hline $20-29$ & 31,80 & 16,58 & 15,22 & $<0,001$ \\
\hline $30-39$ & 26,50 & 16,65 & 9,85 & \\
\hline $40-49$ & 22,78 & 17,62 & 5,16 & \\
\hline $50-59$ & 18,91 & 15,79 & 3,13 & \\
\hline \multicolumn{5}{|l|}{ Escolaridade (anos) } \\
\hline $0-4$ anos & 15,92 & 10,95 & 4,97 & 0,337 \\
\hline 5-8 anos & 14,27 & 10,31 & 3,96 & \\
\hline 9-11 anos & 20,74 & 14,52 & 6,22 & \\
\hline$\geq 12$ anos & 49,07 & 30,86 & 18,21 & \\
\hline \multicolumn{5}{|l|}{ Nível Socioeconômico } \\
\hline $\mathrm{A} / \mathrm{B}$ & 27,33 & 18,86 & 8,47 & 0,489 \\
\hline $\mathrm{C}$ & 65,81 & 42,81 & 23,00 & \\
\hline $\mathrm{D} / \mathrm{E}$ & 6,86 & 5,03 & 1,83 & \\
\hline \multicolumn{5}{|l|}{ Estado Nutricional $\left(\mathrm{kg} / \mathrm{m}^{2}\right)$} \\
\hline Peso Normal $(<25)$ & 53,68 & 30,12 & 23,56 & $<0,001$ \\
\hline Excesso de Peso $(\geq 25)$ & 46,32 & 36,47 & 9,86 & \\
\hline \multicolumn{5}{|l|}{ Perímetro da Cintura $(\mathrm{cm})$} \\
\hline Normal $(<80 \mathrm{M} ;<90 \mathrm{H})$ & 56,32 & 30,48 & 25,83 & $<0,001$ \\
\hline Elevada $(\geq 80 \mathrm{M} ; \geq 90 \mathrm{H})$ & 43,68 & 36,16 & 7,52 & \\
\hline
\end{tabular}

*Significância estatística $(\mathrm{p}<0,05)$. Teste do qui-quadrado de Pearson.

Fonte: Elaborado pelas autoras.

Na Tabela 4, observa-se que os indivíduos com idade entre 30-39 anos, TG e razão TG/HDL normais apresentaram maior ingestão de MUFA ( $\mathrm{p}=0,024, \mathrm{p}=0,015$ e $\mathrm{p}=0,020$, respectivamente). A ingestão média de MUFA foi de 32,20 $\mathrm{g}( \pm 1,96)$ por dia na população, com uma prevalência de $96,6 \%$ de indivíduos ingerindo quantidades abaixo da recomendação. Já a ingestão média de ômega-3 foi o único nutriente que maioria da população $(91,5 \%)$ ingeriu em quantidades recomendadas pela diretriz, acima de $1 \mathrm{~g} / \mathrm{dia}$, obtendo uma média de $1,97 \mathrm{~g}( \pm 0,6)$ por dia. A ingestão adequada de ômega- 3 foi mais frequente entre os indivíduos com o CT normal $(\mathrm{p}=0,015)$.

\section{Discussão}

Comparando-se com outros dados da literatura, a prevalência total encontrada de dislipidemia $(64,25 \%)$ foi mais elevada em relação ao estudo de Souza et al. ${ }^{29}$ em Campos dos Goytacazes, Rio de Janeiro, onde verificaram uma prevalência de $24,2 \%$ de dislipidemia na população e, ao estudo de Fernandes et al. ${ }^{1}$, desenvolvido em oito cidades do estado de São Paulo que observou um percentual $12,2 \%$. A prevalência de dislipidemia da população de Viçosa esteve mais próxima da encontrada por Moraes et al. ${ }^{8}(61,9 \%)$ em estudo desenvolvido em Ribeirão Preto, São Paulo, assim como na pesquisa desenvolvida por Garcez et al. ${ }^{9}$ na população de São Paulo, onde a frequência encontrada foi de $60,3 \%$ entre adultos. Essas diferenças nas prevalências entre os estudos podem ser atribuídas aos pontos de corte distintos utilizados na época das investigações, a idade dos grupos avaliados, assim como ao nível socioeconômico. Além disso, a forma de obtenção do diagnóstico de dislipidemia (autorreferida ou testada) também pode ter interferido nos resultados.

O diagnóstico de dislipidemia por pelo menos um dos parâmetros alterados foi observado, principalmente, entre indivíduos com excesso 
Tabela 2. Consumo de gorduras e carboidratos, segundo a presença de dislipidemias na população adulta (20 a 59 anos: $\mathrm{n}=884$ ) de Viçosa-MG, Brasil (2012/2014).

\begin{tabular}{|c|c|c|c|c|}
\hline \multirow[b]{2}{*}{ Variáveis } & \multirow[b]{2}{*}{$\begin{array}{c}\text { Total } \\
(\%)\end{array}$} & \multicolumn{2}{|c|}{ Dislipidemias } & \multirow[b]{2}{*}{$\mathbf{p}^{*}$} \\
\hline & & $\begin{array}{l}\text { Sim } \\
(\%)\end{array}$ & $\begin{array}{l}\text { Não } \\
(\%)\end{array}$ & \\
\hline \multicolumn{5}{|l|}{$\begin{array}{l}\text { Gordura Saturada } \\
(\%)\end{array}$} \\
\hline Adequado $(<10)$ & 6,53 & 4,55 & 1,98 & 0,633 \\
\hline $\begin{array}{l}\text { Inadequado } \\
(\geq 10)\end{array}$ & 93,47 & 62,09 & 31,38 & \\
\hline \multicolumn{5}{|l|}{ Gordura trans (\%) } \\
\hline Adequado $(<1)$ & 25,80 & 18,24 & 7,57 & 0,191 \\
\hline Inadequado $(\geq 1)$ & 74,20 & 25,79 & 25,79 & \\
\hline \multicolumn{5}{|l|}{ MUFA (\%) } \\
\hline Adequado (>15) & 3,41 & 2,00 & 1,41 & 0,261 \\
\hline $\begin{array}{l}\text { Inadequado } \\
(\leq 15)\end{array}$ & 96,59 & 64,65 & 31,95 & \\
\hline \multicolumn{5}{|l|}{ Ômega 3 (g) } \\
\hline Adequado $(\geq 1)$ & 91,49 & 60,77 & 30,72 & 0,688 \\
\hline $\begin{array}{l}\text { Inadequado } \\
(<1)\end{array}$ & 8,51 & 5,87 & 2,64 & \\
\hline \multicolumn{5}{|l|}{ Carboidratos (g) } \\
\hline $\begin{array}{l}\text { Adequado } \\
(\leq 130)\end{array}$ & 0,67 & 0,34 & 0,33 & 0,295 \\
\hline $\begin{array}{l}\text { Inadequado } \\
(>130)\end{array}$ & 99,33 & 66,30 & 33,03 & \\
\hline
\end{tabular}

Fonte: Elaborado pelas autoras.

de peso, circunferência da cintura elevada e idade entre 40-59 anos. De acordo com Oliveira et al. ${ }^{30}$, indivíduos expostos tanto a excesso de peso quanto a um excesso de gordura abdominal, independente do sexo, coloca-os em condição de maior predisposição para alterações crônicas. Isso porque o acúmulo de tecido adiposo intra -abdominal aumenta o fluxo de ácidos graxos livres transportados para o fígado. Esses ácidos graxos livres em excesso interagem com a secreção das lipoproteínas, modificando os níveis sanguíneos de triglicerídeos e HDL-C ${ }^{31}$.

Segundo a POF 2008-2009, que avaliou uma amostra representativa da população brasileira, a prevalência de inadequação da ingestão de gordura saturada entre adultos foi de $87 \%{ }^{26}$, percentual maior do que o encontrado para a população de Viçosa $(61,2 \%)$. A PNS de 2013 identificou o consumo regular de carnes com excesso de gordura e leite integral para $37,2 \%$ e $62 \%$ da população, respectivamente ${ }^{32}$. O consumo desses alimentos, fontes de gorduras saturadas, foi mais frequente entre homens, jovens e pessoas com menor escolaridade.

Em relação à idade, os adultos entre 20 e 29 anos apresentaram maiores percentuais de inadequação da ingestão de gorduras saturadas, trans e monoinsaturadas. Castro et al. ${ }^{33}$ também identificaram uma menor ingestão de ácidos graxos trans conforme o aumento da idade em seu estudo com adolescentes, adultos e idosos na cidade de São Paulo-SP em 2003, fato que está relacionado a presença de um padrão alimentar rico em alimentos ultraprocessados. Dessa forma, tornam-se necessárias ações de reeducação alimentar e nutricional para adequação da ingestão de gorduras, principalmente em adultos jovens, independente da presença de dislipidemias, prevenindo a ocorrência de agravos no futuro.

No presente estudo, a ingestão elevada de gorduras saturadas e trans foi mais frequente entre indivíduos com escolaridade acima de 12 anos. Entre os participantes da Coorte de Nascimentos de Pelotas, o consumo de ultraprocessados, produtos alimentícios ricos em gorduras saturadas e trans, entre indivíduos que estudaram por 12 anos, foi até 4,8 vezes maior do que aqueles que estudaram por 4 anos. Os autores sugerem uma associação direta entre a maior escolaridade e o consumo de alimentos gordurosos pelo acesso e procura a esses produtos ${ }^{34}$.

Pela mesma razão da escolaridade, acreditase que existe uma relação crescente entre nível socioeconômico e ingestão de gorduras ${ }^{34,35}$, o que não foi observado entre a população de Viçosa, já que apenas a ingestão de gordura trans foi significativamente maior para indivíduos de nível socioeconômico "C". Ainda assim, é importante destacar que no Brasil o consumo de alimentos ricos em gorduras e carboidratos vêm aumentando independentemente da classe social, sobretudo, entre famílias menos favorecidas ${ }^{36}$.

Embora o diagnóstico global da dislipidemia não tenha se associado com as variáveis de consumo, os lipídios séricos quando avaliados por suas razões apresentaram diferenças significativas. A ingestão elevada de gordura saturada e trans foram mais frequentes entre indivíduos com valores normais para CT/HDL-c e TG/HDL -c. Algumas ressalvas podem justificar esse resultado: primeiro, a dislipidemia é uma manifestação que acontece em longo prazo e, considerando que parte da amostra é jovem, pode ser possível ainda não observar essa manifestação clínica provocada pela alimentação inadequada ${ }^{36}$. 
Tabela 3. Variáveis sociodemográficas e bioquímicas segundo o consumo de gordura saturada, trans e carboidratos na população adulta (20 a 59 anos: n=884) de Viçosa-MG, Brasil (2012/2014).

\begin{tabular}{|c|c|c|c|c|c|c|c|c|c|}
\hline \multirow{3}{*}{ Variáveis } & \multicolumn{2}{|c|}{$\begin{array}{l}\text { Gordura } \\
\text { Saturada }\end{array}$} & \multicolumn{3}{|c|}{ Gordura Trans } & \multicolumn{4}{|c|}{ Carboidratos } \\
\hline & $<10 \%$ & $\geq 10 \%$ & $\mathbf{p}^{*}$ & $<1 \%$ & $\geq 1 \%$ & $\mathbf{p}^{*}$ & $\leq 130 \mathrm{~g}$ & $>130 \mathrm{~g}$ & $\mathbf{p}^{*}$ \\
\hline & $(\%)$ & $(\%)$ & & $(\%)$ & $(\%)$ & & $(\%)$ & $(\%)$ & \\
\hline \multicolumn{10}{|l|}{ Sexo } \\
\hline Masculino & 22,30 & 30,00 & 0,374 & 14,47 & 37,84 & 0,568 & 0,26 & 0,41 & 0,497 \\
\hline Feminino & 16,49 & 31,20 & & 11,33 & 36,36 & & 52,05 & 47,29 & \\
\hline \multicolumn{10}{|l|}{ Faixa Etária (anos) } \\
\hline $20-29$ & 7,96 & 23,84 & 0,012 & 4,59 & 27,21 & $<0,001$ & 0,45 & 31,36 & 0,205 \\
\hline $30-39$ & 9,53 & 16,96 & & 5,97 & 20,52 & & 0,11 & 26,38 & \\
\hline $40-49$ & 10,36 & 12,43 & & 6,96 & 15,82 & & 0,00 & 22,78 & \\
\hline $50-59$ & 10,95 & 7,97 & & 8,28 & 10,64 & & 0,10 & 18,81 & \\
\hline \multicolumn{10}{|l|}{ Escolaridade (anos) } \\
\hline $0-4$ & 9,91 & 6,01 & $<0,001$ & 6,42 & 9,51 & 0,009 & 0,00 & 15,92 & 0,670 \\
\hline $5-8$ & 7,02 & 7,24 & & 4,77 & 9,50 & & 0,00 & 14,27 & \\
\hline $9-11$ & 8,41 & 12,33 & & 5,32 & 15,42 & & 0,17 & 20,57 & \\
\hline$\geq 12$ & 13,45 & 35,62 & & 9,30 & 39,77 & & 0,50 & 48,57 & \\
\hline \multicolumn{10}{|l|}{ Nível Socioeconômico } \\
\hline $\mathrm{A} / \mathrm{B}$ & 8,96 & 18,37 & 0,161 & 6,22 & 21,11 & 0,027 & 0,19 & 27,14 & 0,728 \\
\hline $\mathrm{C}$ & 26,64 & 39,17 & & 16,35 & 49,46 & & 0,48 & 65,33 & \\
\hline $\mathrm{D} / \mathrm{E}$ & 3,23 & 3,63 & & 3,26 & 3,60 & & 0,00 & 6,86 & \\
\hline \multicolumn{10}{|l|}{ Estado Nutricional $\left(\mathrm{kg} / \mathrm{m}^{2}\right)$} \\
\hline Peso Normal $(<25)$ & 19,80 & 33,87 & 0,254 & 12,78 & 40,89 & 0,259 & 0,50 & 53,17 & 0,259 \\
\hline Excesso de Peso $(\geq 25)$ & 18,95 & 27,37 & & 12,89 & 33,43 & & 0,17 & 46,16 & \\
\hline \multicolumn{10}{|l|}{ Perímetro da Cintura $(\mathrm{cm})$} \\
\hline Normal $(<80 \mathrm{M} ;<90 \mathrm{H})$ & 18,88 & 37,44 & 0,038 & 12,24 & 44,08 & 0,010 & 0,50 & 55,82 & 0,256 \\
\hline Elevada $(\geq 80 \mathrm{M} ; \geq 90 \mathrm{H})$ & 19,92 & 23,77 & & 13,56 & 30,12 & & 0,17 & 43,52 & \\
\hline \multicolumn{10}{|l|}{ Colesterol Total (mg/dL) } \\
\hline Normal $(<200)$ & 22,72 & 39,23 & 0,327 & 13,66 & 48,29 & 0,068 & 0,67 & 61,28 & 0,004 \\
\hline Elevado $(\geq 200)$ & 16,08 & 21,98 & & 12,15 & 25,91 & & 0,00 & 38,06 & \\
\hline \multicolumn{10}{|l|}{ HDL-c (mg/dL) } \\
\hline Normal $(\geq 40 \mathrm{H} ; \geq 50 \mathrm{M})$ & 17,53 & 22,50 & 0,022 & 10,26 & 29,78 & 0,918 & 59,64 & 39,69 & 0,454 \\
\hline Baixo $(<40 \mathrm{H} ;<50 \mathrm{M})$ & 21,27 & 38,70 & & 15,55 & 44,42 & & 0,33 & 0,34 & \\
\hline \multicolumn{10}{|l|}{ LDL-c (mg/dL) } \\
\hline Normal $(<160)$ & 34,49 & 54,26 & 0,932 & 23,14 & 65,60 & 0,730 & 0,67 & 88,08 & 0,239 \\
\hline Elevado $(\geq 160)$ & 4,31 & 6,95 & & 2,66 & 8,60 & & 0,00 & 11,26 & \\
\hline \multicolumn{10}{|l|}{ Triglicerídeos (mg/dL) } \\
\hline Normal $(<150)$ & 27,43 & 46,16 & 0,270 & 15,41 & 58,19 & 0,001 & 0,56 & 73,03 & 0,539 \\
\hline Elevado $(\geq 150)$ & 11,37 & 15,04 & & 10,39 & 16,01 & & 0,10 & 26,30 & \\
\hline \multicolumn{10}{|l|}{$\mathrm{CT} / \mathrm{HDL}$} \\
\hline Normal $(\leq 5 \mathrm{H} ; \leq 4,5 \mathrm{M})$ & 25,92 & 46,33 & 0,025 & 16,37 & 55,89 & 0,033 & 0,58 & 71,68 & 0,306 \\
\hline Elevado $(>5 \mathrm{H} ;>4,5 \mathrm{M})$ & 12,88 & 14,87 & & 9,44 & 18,31 & & 0,00 & 27,66 & \\
\hline \multicolumn{10}{|l|}{ LDL/HDL } \\
\hline Normal $(\leq 3,5 \mathrm{H} ; \leq 3,0 \mathrm{M})$ & 29,25 & 9,54 & 0,048 & 19,41 & 58,74 & 0,423 & 0,58 & 7,758 & 0,505 \\
\hline Elevado $(>3,5 \mathrm{H} ;>3,0 \mathrm{M})$ & 48,90 & 12,30 & & 6,39 & 15,46 & & 0,00 & 21,76 & \\
\hline \multicolumn{10}{|l|}{ TG/HDL } \\
\hline Normal $(<4)$ & 27,51 & 49,60 & 0,047 & 17,61 & 59,49 & 0,011 & 0,67 & 76,44 & 0,014 \\
\hline Elevado $(\geq 4)$ & 11,29 & 11,61 & & 8,19 & 14,71 & & 0,00 & 22,89 & \\
\hline
\end{tabular}

${ }^{\star}$ Significância estatística $(\mathrm{p}<0,05)$. Teste do qui-quadrado de Pearson.

Fonte: Elaborado pelas autoras. 
Tabela 4. Variáveis sociodemográficas e bioquímicas segundo o consumo de gordura monoinsaturada e ômega-3 na população adulta (20 a 59 anos: $n=884)$ de Viçosa-MG, Brasil (2012/2014).

\begin{tabular}{|c|c|c|c|c|c|c|}
\hline \multirow{3}{*}{ Variáveis } & \multicolumn{2}{|c|}{$\begin{array}{c}\text { Gordura } \\
\text { Monoinsaturada }\end{array}$} & \multicolumn{4}{|c|}{ Ômega 3} \\
\hline & $\leq 15 \%$ & $>15 \%$ & $\mathbf{p}^{*}$ & $\leq 1 \mathrm{~g}$ & $>1 g$ & $\mathbf{p}^{*}$ \\
\hline & $(\%)$ & $(\%)$ & & $(\%)$ & $(\%)$ & \\
\hline \multicolumn{7}{|l|}{ Sexo } \\
\hline Masculino & 50,82 & 1,49 & 0,282 & 3,38 & 48,93 & 0,111 \\
\hline Feminino & 45,77 & 1,92 & & 5,13 & 42,56 & \\
\hline \multicolumn{7}{|l|}{ Faixa Etária (anos) } \\
\hline $20-29$ & 31,10 & 0,71 & 0,024 & 2,17 & 29,63 & 0,179 \\
\hline $30-39$ & 25,22 & 1,27 & & 2,12 & 24,37 & \\
\hline $40-49$ & 21,71 & 1,08 & & 1,37 & 21,42 & \\
\hline $50-59$ & 18,57 & 0,35 & & 2,85 & 16,06 & \\
\hline \multicolumn{7}{|l|}{ Escolaridade (anos) } \\
\hline $0-4$ & 15,81 & 0,11 & 0,313 & 1,37 & 14,56 & 0,943 \\
\hline $5-8$ & 13,70 & 0,57 & & 1,14 & 13,13 & \\
\hline $9-11$ & 20,04 & 0,70 & & 1,54 & 19,19 & \\
\hline$\geq 12$ & 47,05 & 2,02 & & 4,47 & 44,61 & \\
\hline \multicolumn{7}{|l|}{ Nível Socioeconômico } \\
\hline $\mathrm{A} / \mathrm{B}$ & 26,15 & 1,17 & 0,618 & 1,75 & 25,58 & 0,197 \\
\hline $\mathrm{C}$ & 63,87 & 1,94 & & 5,61 & 60,20 & \\
\hline $\mathrm{D} / \mathrm{E}$ & 6,57 & 0,30 & & 1,16 & 5,70 & \\
\hline \multicolumn{7}{|l|}{ Estado Nutricional $\left(\mathrm{kg} / \mathrm{m}^{2}\right)$} \\
\hline Peso Normal $(<25)$ & 51,75 & 1,92 & 0,794 & 4,54 & 49,14 & 0,934 \\
\hline Excesso de Peso $(\geq 25)$ & 44,84 & 1,49 & & 3,99 & 42,34 & \\
\hline \multicolumn{7}{|l|}{ Perímetro da Cintura $(\mathrm{cm})$} \\
\hline Normal $(<80 \mathrm{M} ;<90 \mathrm{H})$ & 54,31 & 2,01 & 0,799 & 4,73 & 51,59 & 0,897 \\
\hline Elevada $(\geq 80 \mathrm{M} ; \geq 90 \mathrm{H})$ & 42,28 & 1,40 & & 3,78 & 39,90 & \\
\hline \multicolumn{7}{|l|}{ Colesterol Total (mg/dL) } \\
\hline Normal $(<200)$ & 59,67 & 2,27 & 0,618 & 3,93 & 58,01 & 0,015 \\
\hline Elevado $(\geq 200)$ & 36,92 & 1,14 & & 4,58 & 33,48 & \\
\hline \multicolumn{7}{|l|}{ HDL-c (mg/dL) } \\
\hline Normal $(\geq 40 \mathrm{H} ; \geq 50 \mathrm{M})$ & 38,88 & 1,16 & 0,464 & 2,78 & 37,25 & 0,223 \\
\hline Baixo $(<40 \mathrm{H} ;<50 \mathrm{M})$ & 57,72 & 2,25 & & 5,73 & 54,24 & \\
\hline \multicolumn{7}{|l|}{ LDL-c (mg/dL) } \\
\hline Normal $(<160)$ & 85,68 & 3,06 & 0,848 & 7,54 & 81,20 & 0,961 \\
\hline Elevado $(\geq 160)$ & 10,91 & 0,35 & & 0,97 & 10,29 & \\
\hline \multicolumn{7}{|l|}{ Triglicerídeos (mg/dL) } \\
\hline Normal $(<150)$ & 70,41 & 3,18 & 0,015 & 6,30 & 67,30 & 0,951 \\
\hline Elevado $(\geq 150)$ & 26,18 & 0,23 & & 2,21 & 24,19 & \\
\hline \multicolumn{7}{|l|}{ CT/HDL } \\
\hline Normal $(\leq 5 \mathrm{H} ; \leq 4,5 \mathrm{M})$ & 69,52 & 2,73 & 0,315 & 6,11 & 66,15 & 0,941 \\
\hline Elevado $(>5 \mathrm{H} ;>4,5 \mathrm{M})$ & 27,07 & 0,68 & & 2,40 & 25,34 & \\
\hline \multicolumn{7}{|l|}{$\mathrm{LDL} / \mathrm{HDL}$} \\
\hline $\operatorname{Normal}(\leq 3,5 \mathrm{H} ; \leq 3 \mathrm{M})$ & 75,58 & 21,01 & 0,637 & 6,69 & 71,46 & 0,936 \\
\hline Elevado $(>3,5 \mathrm{H} ;>3 \mathrm{M})$ & 2,57 & 0,83 & & 1,82 & 20,03 & \\
\hline \multicolumn{7}{|l|}{ TG/HDL } \\
\hline Normal $(<4)$ & 73,82 & 3,28 & 0,020 & 6,63 & 70,48 & 0,899 \\
\hline Elevado $(\geq 4)$ & 22,77 & 0,12 & & 1,88 & 21,01 & \\
\hline
\end{tabular}

${ }^{\star}$ Significância estatística $(\mathrm{p}<0,05)$. Teste do qui-quadrado de Pearson.

Fonte: Elaborado pelas autoras. 
Segundo pesquisas realizadas no Brasil, mesmo quando os indivíduos recebem o diagnóstico da dislipidemia, podem não aderir à hábitos saudáveis (alimentação, atividade física, farmacoterapia) e tendem a buscar assistência somente quando ocorrem complicações clínicas graves ${ }^{37}$.

Nesse estudo, os indivíduos com HDL-c baixo e razão LDL/HDL elevada possuíram prevalência significativamente maior da ingestão de gorduras saturadas acima de $10 \%$ do VCT. Para Mensink et al. ${ }^{38}$, o efeito das gorduras saturadas no colesterol HDL deve ser interpretado com cautela, já que depende muito mais do tipo do ácido graxo saturado consumido (tamanho da cadeia carbônica) e da proporção dos outros nutrientes da dieta. Ao substituir as gorduras saturadas por gorduras monoinsaturadas ou ácido láurico, foi observado aumento do HDL-c. Quando as gorduras saturadas foram substituídas por carboidratos ou pelos demais ácidos graxos de cadeia longa, o efeito no HDL-c foi inverso.

Souza et al. $^{39}$, em sua revisão sistemática, identificaram que gorduras trans têm uma relação estabelecida com mortalidade global, Doenças Cardiovasculares (DCV) e diabetes tipo 2, independentemente de outros fatores. Cabe ressaltar que o mesmo não ocorre para as gorduras saturadas, cuja relação com DCV é inconclusi$\mathrm{va}^{40}$. Durante décadas, as recomendações foram de dietas com baixo teor de gordura saturada para a prevenção das DCV, mas o efeito desse componente deve ser avaliado juntamente com os outros nutrientes que compõem a dieta, e não de forma isolada, sendo a análise do padrão alimentar mais apropriado para investigar sua relação com DCV ${ }^{41,42}$.

A ingestão de carboidratos também foi elevada, independentemente da presença de dislipidemia. A média de ingestão de carboidratos foi aproximadamente $343 \mathrm{~g}$, o que corresponde a aproximadamente $164 \%$ de ingestão acima da recomendação que é $130 \mathrm{~g}$. Esse valor esteve muito acima das quantidades obtidas na POF 20082009, que identificou média de consumo nos indivíduos com 19 a 59 anos entre 240 e 290 g para mulheres e homens, respectivamente. As dietas hiperglicídicas, com carboidratos geralmente provenientes de alimentos processados e de alto índice glicêmico, contribuem para a hipertrigliceridemia, favorecendo a maior formação de partículas de LDL pequenas e densas, e redução das concentrações de HDL-c plasmático ${ }^{43}$.

A ingestão de gorduras monoinsaturadas esteve mais inadequada em indivíduos com TG séricos e com razão TG/HDL-c normais. A razão TG/HDL-c já foi validada em uma amostra da população de Viçosa e obteve uma boa correlação com outros desfechos de saúde desfavoráveis $^{44}$. Apesar desse resultado, pode-se dizer que esses indivíduos também estão expostos a maior risco por aderirem a uma dieta de baixo teor de ácidos graxos monoinsaturados, pois, a ingestão moderada de MUFA é capaz de reduzir os triglicerídeos séricos e aumentar o HDL-c ${ }^{45}$. Um ensaio clínico observou que a substituição de cada $1 \%$ de energia proveniente das gorduras trans por MUFA é capaz de reduzir a razão CT/HDL-c em $0,54^{46}$.

A ingestão de ômega-3 apresentou prevalência de adequação superior entre aqueles com colesterol total abaixo de $200 \mathrm{mg} / \mathrm{dl}$. Sugere-se que redução do colesterol total é um benefício dos ácidos graxos $\omega-3^{47}$. Embora as prevalências para os demais marcadores não tenham diferido na amostra, o $\omega$-3 provoca mecanismos sistêmicos e intracelulares responsáveis pela redução dos triglicerídeos séricos e aumento modesto no HDL-c. Possui, também, propriedades anti -inflamatória, antimicrobiana e vasodilatadora, culminando numa proteção cardiovascular e aterosclerótica ${ }^{48}$.

Dentre as limitações deste estudo, destaca-se o desenho transversal, que não permite estabelecer relação temporal entre as variáveis. Além disso, destaca-se o uso do QFCA, que apesar de ser um método altamente eficaz que apresenta boa correlação com a ingestão verdadeira dos indivíduos, possui a limitação do viés de memória $^{49}$. Como forma de diminuir os efeitos desta limitação, um álbum fotográfico de alimentos foi apresentado aos entrevistados, durante o relato do QFCA, para auxiliá-los a estimar com maior precisão as porções usualmente consumidas.

Conclui-se que nessa amostra representativa da população de Viçosa-MG, Brasil, há uma elevada prevalência de dislipidemias, ingestão excessiva de gorduras saturadas, gorduras trans e carboidratos, além da ingestão de gorduras monoinsaturadas abaixo do ideal. A ingestão elevada de gordura saturada e trans foi maior entre os indivíduos com HDL-c e razões TG/HDL, CT/ HDL e LDL/HDL elevadas, e os indivíduos com colesterol total normal possuíam maior percentual de adequação da ingestão de ômega-3. São necessários mais estudos para elucidar a associação entre a ingestão de gorduras e carboidratos e as alterações dos lipídios séricos na população. 


\section{Colaboradores}

SEO Valença e ADM Brito: concepção, delineamento, análise e interpretação dos dados e redação do artigo. DCG Silva: planejamento, concepção, delineamento, análise e interpretação dos dados e redação do artigo. FG Ferreira e JF Novaes: redação e revisão crítica do artigo. GZ Longo: planejamento, coleta de dados e revisão crítica do artigo. Todas as autoras leram e aprovaram a versão final do manuscrito.

\section{Agradecimentos}

As autoras agradecem a todos os voluntários que contribuíram para o estudo.

Agradecemos também ao Conselho Nacional de Desenvolvimento Científico e Tecnológico $(\mathrm{CNPq})$, a BIOCLIN e à Fundação de Amparo à Pesquisa do Estado de Minas Gerais (FAPEMIG) pelo financiamento do projeto.

\section{Referências}

1. Fernandes RA, Christofaro DGD, Casonatto J, Codogno JS, Rodrigues EQ, Cardoso ML, Kawaguti SS, Zanesco A. Prevalence of Dyslipidemia in Individuals Physically Active During Childhood, Adolescence and Adult Age. Arq Bras Cardiol 2011; 97(4):317-323.

2. Siri PW, Krauss RM. Influence of Dietary Carbohydrate and Fat on LDL and HDL Particle Distributions. Curr Atheroscler Rep 2005; 7(6):455-459.

3. Sociedade Brasileira de Cardiologia (SBC). I Diretriz sobre o Consumo de Gorduras e Saúde Cardiovascular. Arq Bras Cardiol 2013; 100(1):1-40.

4. Herrington W, Lacey B, Sherliker P, Armitage J, Lewington S. Epidemiology of Atherosclerosis and the Potential to Reduce the Global Burden of Atherothrombotic Disease. Circ Res 2016; 118(4):535-546.

5. Ribeiro ALP, Duncan BB, Brant LCC, Lotufo PA, Mill JG, Barreto SM. Cardiovascular Health in Brazil: Trends and Perspectives. Circulation 2016; 133(4):422-433.

6. Barquera S, Pedroza-Tobías A, Medida C, Hernandez-Barrera L, Bibbins-Domingo K, Lozano R, Moran AE. Global Overview of the Epidemiology of Atherosclerotic Cardiovascular Disease. Arch Med Res 2015; 46(5):328-338.

7. Marinho F, Passos VMA, França EB. Novo século, novos desafios: mudança no perfil da carga de doença no Brasil de 1990 a 2010. Epidemiol Serv Saude 2016; 25(4):713-724.

8. Moraes VER, Checchio MV, Freitas, ICM. Dislipidemia e fatores associados em adultos residentes em Ribeirão Preto, SP: resultados do Projeto EPIDCV. Arq Bras Endocrinol Metabol 2013; 57(9):691-701.

9. Garcez MR, Pereira JL, Fontanelli MM, Marchioni DML, Fisberg RM. Prevalence of dyslipidemia according to the nutritional status in a representative sample of São Paulo. Arq Bras Cardiol 2014; 103(6):476484.

10. Loureiro NSL, Amaral TLM, Amaral CA, Monteiro GTR, Vasconcellos MTL, Bortolini MJS. Relação de indicadores antropométricos com fatores de risco para doença cardiovascular em adultos e idosos de Rio Branco, Acre. Rev Saude Publica 2020; 54:24.

11. Instituto Brasileiro de Geografia e Estatística (IBGE). Pesquisa Nacional de Saúde 2013. Percepção do estado de saúde. Estio de vida e doenças crônicas. Rio de Janeiro: IBGE; 2014.

12. Brasil. Ministério da Saúde (MS). Vigitel Brasil 2016 Saúde Suplementar: vigilância de fatores de risco e proteção para doenças crônicas por inquérito telefônico. Brasília: MS; 2017.

13. Estruch R. Mortalidad cardiovascular: como prevenirla? Nefrología 2014; 34(5):561-569.

14. Dearborn JL, Urrutia VC, Kernan WN. The Case for Diet: A Safe and Efficacious Strategy for Secondary Stroke Prevention. Front Neurol 2015; 6(2):1-8.

15. Otto MCO, Afshin A, Micha R, Khatibzades S, Fahimi S, Singh G, Danaei G, Monteiro CA, Louzada MLC, Ezzati M, Mozaffarian D. The Impact of Dietary and Metabolic Risk Factors on Cardiovascular Diseases and Type 2 Diabetes Mortality in Brazil, Global Burden of Diseases, Injuries, and Risk Factors Metabolic Risk Factors of Chronic Diseases Expert Group and Nutrition and Chronic D. PloS One 2016; 11(3):e0151503. 
16. Silva DCG, Segheto W, Lima MFC, Pessoa MC, Peluzio MCG, Marchioni DML, Cunha DB, Longo GZ. Using the method of triads in the validation of a food frequency questionnaire to assess the consumption of fatty acids in adults. J Hum Nutr Die 2018; 31(1):8595.

17. Segheto W, Silva DCG, Coelho FA, Reis VG, Morais SHO, Marins JCB, Ribeiro AQ, Longo GZ. Body adiposity index and associated factors in adults: method and logistics of a population-based study. Nutr Hosp 2015; 32(1):101-109.

18. Associação Brasileira de Empresas de Pesquisa (ABEP). Critério de classificação econômica Brasil Dados com base no levantamento socioeconômico. Rio de Janeiro: IBOPE; 2012

19. World Health Organization (WHO). Preventing and managing the global epidemic: Report of a WHO Consultation. Genebra: WHO; 2000.

20. International Diabetes Federation (IDF). The IDF consensus worldwide definition of the metabolic syndrome. Bruxelas: IDF; 2006.

21. Friedewald WT, Levy RI, Fredrickson DS. Estimation of the concentration of low-density lipoprotein cholesterol in plasma, without use of the preparative ultracentrifuge. Clin Chem 1972; 18(6):499-502.

22. Bhalodkar NC, Blum S, Enas EA. Accuracy of the Ratio of Triglycerides to High-Density Lipoprotein Cholesterol for Predicting Low-Density Lipoprotein Cholesterol Particle Sizes, Phenotype B, and Particle Concentrations Among Asian Indians. JAMA Cardiol 2006; 97(7):1007-1009.

23. Millán J, Pintó X, Muñoz A, Zúñiga M, Rubiés-Prat J, Pallardo LF, Masana L, Mangas A, Hernández-Mijares A, González-Santos P, Ascaso JF, Pedro-Botet J. Lipoprotein ratios: Physiological significance and clinical usefulness in cardiovascular prevention. Vasc Health Risk Manag 2009; 5:757-765.

24. Sociedade Brasileira de Cardiologia (SBC). Atualização da Diretriz Brasileira de Dislipidemias e Prevenção da Aterosclerose - 2017. Arq Bras Cardiol 2017; 109(2 Supl. 1):1-76.

25. Barufaldi LA, Abreu GA, Veiga GV, Sichieri R, Kuschnir MCC, Cunha DB, Pereira RA, Bloch KV. Programa para registro de recordatório alimentar de 24 horas: aplicação no Estudo de Riscos Cardiovasculares em Adolescentes. Rev Bras Epidemiol 2016; 19(2):464468.

26. Instituto Brasileiro de Geografia e Estatística (IBGE). Pesquisa de Orçamentos Familiares 2008-2009: Análise do Consumo Alimentar Pessoal no Brasil. Rio de Janeiro: IBGE; 2011.

27. Andrade GA, Pereira RA, Sichieri R. Consumo alimentar de adolescentes com e sem sobrepeso do $\mathrm{Mu}$ nicípio do Rio de Janeiro. Cad Saude Publica 2003; 9(5):1485-1495.

28. Institute of Medicine (IOM). Dietary Reference Intakes for Energy, Carbohydrate, Fiber, Fat, Fatty Acids, Cholesterol, Protein, and Amino Acids (Macronutrients). Washington, D.C.: The National Acadmies Press; 2005.
29. Souza LJ, Filho JTDS, Souza TF, Reis AFF, Neto CG, Bastos DA, Côrtes VA, Chalita FEB, Teixeira CL. Prevalência de Dislipidemia e Fatores de Risco em Campos dos Goytacazes - RJ. Arq Bras Cardiol 2003; 81(3):249-256

30. Oliveira LPM, Assis AMO, Silva MCM, Santana MLP Santos NS, Pinheiro SMC, Barreto ML, Souza CO. Fatores associados a excesso de peso e concentração de gordura abdominal em adultos na cidade de Salvador, Bahia, Brasil. Cad Saude Publica 2009; 25(3):570-582.

31. Ebbert J, Jensen M. Fat Depots, Free Fatty Acids, and Dyslipidemia. Nutrients 2013; 5(2):498-508.

32. Claro RM, Santos MAS, Oliveira TPO, Pereira CA, Szwarcwald CL, Malta DC. Consumo de alimentos insalubres relacionados a enfermidades crónicas no transmisibles en Brasil: Encuesta Nacional de Salud, 2013. Epidemiol Serv Saude 2015; 24(2):257-265.

33. Castro MA, Barros RR, Bueno MB, César CLG, Fisberg RM. Trans fatty acid intake among the population of the city of São Paulo, Brazil. Rev Saude Publica 2009; 43(6):991-997.

34. Bielemann RM, Motta JVS, Minten GC, Horta BL, Gigante DP. Consumo de alimentos ultra processados e impacto na dieta de adultos jovens. Rev Saude Publica 2015; 49(28):1-10.

35. Levy RB, Claro RM, Mondini L, Sichieri R, Monteiro CA. Distribuição regional e socioeconômica da disponibilidade domiciliar de alimentos no Brasil em 20082009. Rev Saude Publica 2012; 46(1):6-15.

36. Martins APB, Levy RB, Claro RM, Moubarac JC, Monteiro CA. Increased contribution of ultra-processed food products in the Brazilian diet (1987-2009). Rev Saude Publica 2013; 47(4):656-665.

37. Secretaria de Saúde do Estado de São Paulo. Prevenção de Doenças Crônicas Não Transmissiveis (DCNT) e de seus Fatores de Risco. São Paulo: SES/CVE/DDCNT, 2008.

38. Mensink RP, Zock PL, Kester AD, Katan MB. Effects of dietary fatty acids and carbohydrates on the ratio of serum total to HDL cholesterol and on serum lipids and apolipoproteins: a meta-analysis of 60 controlled trials. Am J Clin Nutr 2003; 77(5):1146-1155.

39. Souza RJ, Mente A, Maroleanu A, Cozma AI, Ha V, Kishibe T, Uleryk E, Budylowski P, Schünemann H, Beyene J, Anand SS. Intake of saturated and trans unsaturated fatty acids and risk of all cause mortality, cardiovascular disease, and type 2 diabetes: systematic review and meta-analysis of observational studies. BMJ 2015; 351:h3978.

40. Siri-Tarino PW, Sun Q, Hu FB, Krauss RM. Meta-analysis of prospective cohort studies evaluating the association of saturated fat with cardiovascular disease. Am J Clin Nutr 2010; 91(3):535-546.

41. Lottenberg AMP. Importance of the dietary fat on the prevention and control of metabolic disturbances and cardiovascular disease. Arq Bras Endocrinol Metab 2009; 5353(55):595-607.

42. Mozaffarian D, Aro A, Willet WC. Health effects of trans-fatty acids: experimental and observational evidence. Eur J Clin Nutr 2009; 63:S5-S21. 
43. Polacow VO, Lancha AHJ. Dietas Hiperglicídicas: Efeitos da Substituição Isoenergética de Gordura por Carboidratos Sobre o Metabolismo de Lipidios, Adiposidade Corporal e Sua Associação com Atividade. Arq Bras Endocrinol Metab 2007; 53(3):389-400.

44. Martins MV, Souza JD, Martinho KO, Franco FS, Tinôco ALA. Association between triglycerides and HDL-cholesterol ratio and cardiovascular risk factors among elderly persons receiving care under the family health strategy of Viçosa, Minas Gerais. Rev Bras Geriatr Gerontol 2017; 20(2):236-243.

45. Baum SJ, Kris-Etherton PM, Willett WC, Lichtenstein AH, Rudel LL, Maki KC, Whelan J, Ramsden CE, Block RC. Fatty acids in cardiovascular health and disease: A comprehensive update. J Clin Lipidol 2012; 6(3):216-234.

46. Mozaffarian D, Clarke R. Quantitative effects on cardiovascular risk factors and coronary heart disease risk of replacing partially hydrogenated vegetable oils with other fats and oils. Eur J Clin Nutr 2009; 63(S2):S22-S33.

47. Jain AP, Aggarwal KK, Zhang P-Y. Omega-3 fatty acids and cardiovascular disease. Eur Ver Med Pharmacol Sci 2015; 19(3):441-445.

48. Raposo HF. Efeito dos ácidos graxos n-3 e n-6 na expressão de genes do metabolismo de lipídeos e risco de aterosclerose. Rev Nutr 2010; 23(5):871-879.

49. Costa AGV, Priore SE, Sabarense CM, Franceschini SCC. Questionário de frequência de consumo alimentar e recordatório de 24 horas: aspectos metodológicos para avaliação da ingestão de lipídeos. Rev Nutr 2006; 19(5):631-641.

Artigo apresentado em 28/05/2020

Aprovado em 15/09/2020

Versão final apresentada em 17/09/2020

Editores-chefes: Romeu Gomes, Antônio Augusto Moura da Silva 